\title{
MULTI-DIMENSIONAL INTEGRAL INEQUALITIES OF THE WIRTINGER-TYPE
}

\author{
WING-SuM CHEUng AND JOSIP PEČARIĆ
}

Abstract. In this paper some new Wirtinger-type integral inequalities involving many functions of many variables are established. These on the one hand improve existing results in the subject concerned and on the other hand can serve as generators of other integral inequalities of such type.

Mathematics subject classification (1991): 26D10, 26D15, 39B72.

Key words and phrases: Wirtinger's inequality.

\section{REFERENCES}

[1] R. P. Agarwal and P. Y. H. PANG,, Opial Inequalities with Applications in Differential and Difference Equations, Kluwer Academic Publishers, Dordrecht, 1995.

[2] R. P. AgARWAL, J. PEČARIĆ AND I. BRNETIĆ, Improved Integral Inequalities in $n$ Independent Variables, Computers Math. Appl. 33, no. 8 (1997), 27-38.

[3] R. P. Agarwal AND Q. SHeng, Sharp integral inequalities in $n$ independent variables, Nonlinear Analysis 26, no. 2 (1996), 179-210.

[4] E. Almansi, Sopra una delle esperienze di Plateau, Ann. Mat. Pura Appl. (3) 12 (1905), 1-17.

[5] P. R. BEESACK, Extensions of Wirtinger's inequality, Trans. Roy. Soc. Canada Sect. III (3) 53 (1959), 21-30.

[6] _ Integral inequalities of the Wirtinger type, Duke Math. J. 25 (1958), 477-498.

[7] R. Bellman, A note on an inequality of E. Schmidt, Bull. Amer. Math. Soc. 50 (1944), 734-736.

[8] W. BlasKChKe, Kreis und kugel, Leipzig, 1916.

[9] W. S. CHEung, On integral inequalities of the Sobolev type, Aeq. Math. 49 (1995), 153-159.

[10] On Poincaré-type integral inequalities, Proc. Amer. Math. Soc. 119, no. 3 (1993), 857-863.

[11] On some new integrodifferential inequalities of the Gronwall and Wendroff type, J. Math. Anal. Appl. 178, no. 2 (1993), 438-449.

[12] Opial-type inequalities with $m$ functions in $n$ variables, Mathematika 39 (1992), 319-326.

[13] G. H. Hardy, J. E. LitTlewood, And G. Polya, Inequalities, Cambridge Univ. Press, Cambridge, 1952.

[14] G. V. Milovanović, D. S. Mitrinović And Th. M. Rassias, Topics in Polynomials: Extremal problems, Inequalities, Zeros, World Scientific Publ. Co., 1994.

[15] D. S. MitrinOvić, Analytic Inequalities, Springer-Verlag, New York, 1970.

[16] B. SZ.-NAGY, Über Integralungleichungen zwischen einer Funktion und ihrer Ableitung, Acta Sci. Math. (Szeged) 10 (1941), 67-74.

[17] Z. OPIAL, Sur une inégalité, Ann. Polon. Math. 8 (1960), 29-32.

[18] B. G. Pachpatte, On Opial type inequalities in two independent variables, Proc. Royal Soc. Edin. 100A (1985), 263-270.

[19] _ On Poincaré type integral inequalities, J. Math. Anal. Appl. 114 (1986), 111-115.

[20] $\ldots$ On some new integral inequalities in several independent variables, Chinese J. Math. 14, no. 2 (1986), 69-79.

[21] TH. M. RASSIAS, On certain properties of eigenvalues and the Poincaré Inequality, Global Analysis Analysis on Manifolds, Teubner-Texte zur Math, Teubner Leipzig, Band 57 (1983), 282-300. 
[22] Un contre-exemple à l'inégalité de Poincaré, C. R. Acad. Sciences Paris 284 (1977), 409-412.

[23] E. SCHMIDT, Über die Ungleichung, welche die Integrale über eine Potenz einer Funktion und über eine andere Potenz ihrer Ableitung verbindet, Math. Ann. 117 (1940), 301-326. 